\title{
The resonant electromagnetic fields of an array of metallic slits acting as Fabry-Perot cavities
}

\author{
Alastair P. Hibbins, ${ }^{\text {a) }}$ Matthew J. Lockyear, and J. Roy Sambles \\ Electromagnetic Materials Group, School of Physics, University of Exeter, Exeter EX4 4QL, \\ United Kingdom
}

(Received 21 December 2005; accepted 20 March 2006; published online 20 June 2006)

\begin{abstract}
Fabry-Perot cavities are perhaps the best known of the optical transmission resonators, with cavity field enhancement accomplished by two parallel and partially reflecting planes. Recently it has been shown that arrays of narrow slits cut into a metal substrate are similarly able to exhibit resonant transmission modes. An analysis of the field solutions and transmission properties of this resonant array is compared to the well-known etalon and dielectric slab geometries, revealing a most elegant illustration of the principles of Maxwell's electromagnetism. It is demonstrated that the matching of the propagating field to each slit-cavity mode is made possible through strong diffraction at each end. Furthermore, the interface between the slit cavities and semi-infinite space beyond acts as a high-impedance surface on resonance, reflecting the field with a positive reflection-amplitude coefficient. Metallic slit arrays have several advantages over conventional Fabry-Perot resonators with interesting application potential. (C) 2006 American Institute of Physics.
\end{abstract}

[DOI: 10.1063/1.2204818]

\section{INTRODUCTION}

The optical resonant cavity ("etalon") proposed in 1899 by Fabry and Perot ${ }^{1}$ consists of two thin metal films separated by a dielectric and supporting a series of quantized standing wave modes. Transmission maxima associated with these standing wave modes are exhibited on condition that the optical distance between the two reflecting faces equals an integer number of half wavelengths, or

$$
\lambda_{N}=\frac{2 n L}{N},
$$

where $\lambda_{N}$ is the vacuum wavelength of the $N$ th order mode, $n$ is the refractive index, and $L$ is the thickness of the medium separating the two planes. In addition, resonant modes can also be established simply in a high index dielectric slab bounded by air, and the interesting optical field solutions associated with both this and the etalon structure will be described in due course. However the principal focus of this study is the realization that a slit of subwavelength width cut into a metal substrate is also able to support Fabry-Perot (FP) resonant modes, and a periodic array of such elements demonstrates very similar transmission characteristics to those of an etalon. The structure is able to filter radiation in this way because each slit, no matter how narrow, supports a transverse electromagnetic (TEM) waveguide mode. ${ }^{2}$ With the face of the slit array being predominantly metal filled, it will reflect nearly all the radiation incident upon it. Nevertheless weak coupling to the TEM mode in the slits will occur. Reciprocally the mode within each slit cavity will be unable to radiate efficiently, instead being almost perfectly reflected at each end. Hence an open-ended slit will support a series of standing wave modes, and it is the excitation of these modes

${ }^{a)}$ Electronic mail: a.p.hibbins@ex.ac.uk that provides strong resonant enhancement to the otherwise weak transmission channel. The series of resonant transmission peaks has been predicted and experimentally measured by a number of authors; ${ }^{3-10}$ however, a comprehensive study of the boundary conditions and electromagnetic fields associated with these resonant modes is lacking. By calculating the field solutions using a technique based on the finite element method ${ }^{11}$ and by considering the fundamental resonance of the cavity (the boundary conditions for higher order modes are essentially unchanged), we show that the matching of the propagating field to each slit-cavity mode is achieved through strong diffraction at each end of the slit. Furthermore we demonstrate that the interfaces between the slit cavities and semi-infinite space beyond reflect the field with a positive reflection-amplitude coefficient on resonance.

\section{THE ETALON AND DIELECTRIC SLAB FABRY-PEROT CAVITIES}

It is interesting and useful to first discuss the field characteristics of the more familiar forms of FP cavities. Let us begin with the resonant cavity proposed as an interferometer in 1899 (Ref. 1) [Fig. 1(a)]. Between the two metal films a standing wave may be established, having the approximate form

$$
\underline{\mathrm{E}}(z, t)=E_{y} \underline{\hat{y}}=E_{\max }(\sin k z \cos \omega t) \underline{\hat{y}},
$$

where $\omega=2 \pi c / \lambda$ and $k=2 \pi / \lambda$. Since $E_{x}, E_{z}$, and their derivatives, and also $\partial E_{y} / \partial x$, are all zero then Faraday's law, $\underline{\nabla} \times \underline{\mathrm{E}}=-\mu \partial \underline{\mathrm{H}} / \partial t$, reduces to

$$
\frac{\partial E_{y}}{\partial z} \sim \frac{1}{\lambda} H_{x}
$$

Hence the magnetic component of the standing wave is described by 

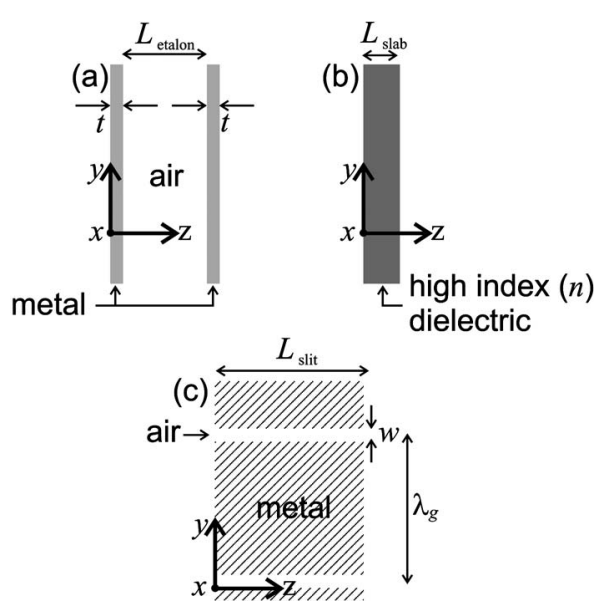

FIG. 1. Schematic diagrams of the (a) etalon, (b) dielectric slab, and (c) metallic slit array sample geometries. In each case, radiation is incident parallel to the $z$ axis and polarized with the electric vector parallel to the $y$ axis.

$$
\underline{\mathrm{H}}(z, t)=H_{x} \underline{\hat{x}}=H_{\max }(\cos k z \sin \omega t) \underline{\hat{x}},
$$

and as one expects for a standing wave, the maximal field enhancements of the electric and magnetic components are separated both spatially and temporally by one-quarter of a cycle (i.e., $\lambda / 4$ and $\tau / 4$, respectively).

Equations (2) and (4) are only precise in the ideal limit of perfectly conducting films into which the electromagnetic fields do not penetrate (i.e., they are totally reflected). However this condition clearly renders coupling to the cavity mode impossible! So consider instead two penetrable metal films, $t=35 \mathrm{~nm}$ thick separated by $L_{\text {etalon }}=265 \mathrm{~nm}$ of air $(n$ $=1$ ). The relative permittivity of the metal is chosen to be $\varepsilon_{r}=-25+0 i$ (with relative permeability $\mu_{r}=1+0 i$ ), which for simplicity is assumed wavelength independent but similar to that of silver at visible frequencies. ${ }^{12}$ The calculated transmission spectrum through the cavity is shown in the inset of Fig. 2. (Radiation is incident parallel to the $z$ axis, i.e., nor-

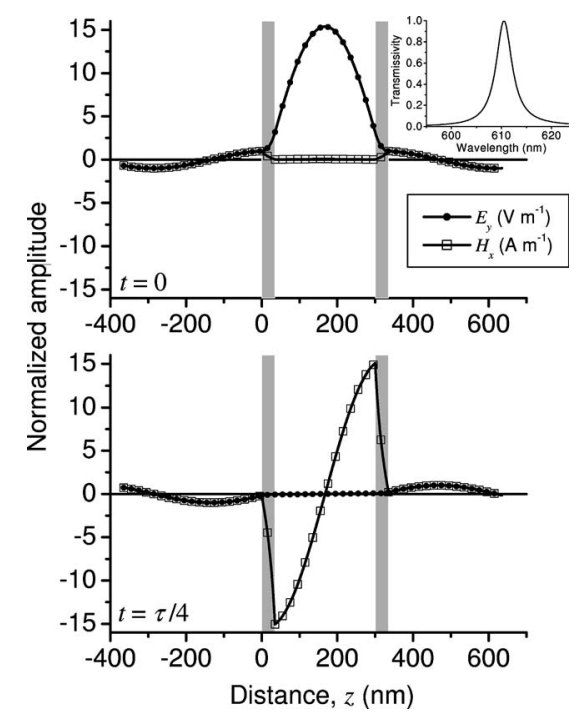

FIG. 2. Predictions of the amplitude of the electric and magnetic fields (normalized to the amplitude of the incident field) through the etalon structure $\left(L_{\text {etalon }}=265 \mathrm{~nm} ; t_{\text {metal }}=35 \mathrm{~nm} ; \varepsilon_{\text {metal }}=-25+0 i\right)$ on resonance of the fundamental mode. Inset: Modeled transmissivity spectrum. mal incidence, and its electric field vector is parallel to the $y$ axis.) There is a strong peak in the transmitted intensity corresponding to the resonance of the fundamental $(N=1)$ mode; however, note that the substitution of the resonant wavelength $(\sim 610 \mathrm{~nm})$ back into Eq. (1) yields a value of $L_{\text {etalon }}$ greater than $265 \mathrm{~nm}$. This increase in the apparent etalon length is due to the aforementioned penetration of the fields into the finite conductivity metal layers, for it is over this distance that the matching of the propagating and cavity fields must take place. The incident polarization dictates that the only nonzero field components in this geometry are $E_{y}$ and $H_{x}$, and these are calculated at the resonant frequency of the fundamental mode (Fig. 2). The fields in the upper graph have been calculated at a time in their cycle associated with the maximum enhancement of the electric field within the cavity. This condition corresponds to a temporal phase, defined as $t=0$, at which a peak of the incident electric field magnitude occurs at the first (illuminated) mirror. Similarly the lower graph shows the same components a quarter of a cycle later $(t=\tau / 4$, where $\tau$ is the temporal period) corresponding to the maximum enhancement of the magnetic field within the cavity and a minimum in the incident magnetic field magnitude at the illuminated face. Hence the cavity modes are driven by the electric component of the incident field. Furthermore, the positions of maximum enhancements are spatially separated along the $z$ axis by a distance equivalent to a quarter of a cycle $(\lambda / 4)$ as expected for an electromagnetic standing wave. The boundary conditions on the metal dictate that while the magnetic field inside the cavity is reflected almost completely in phase, the electric field experiences a near $\pi$-radians phase change on reflection. Hence the peak in the electric field must occur at the cavity center and that of the magnetic field occurs at the metal surface. Since the energy stored in a standing wave is equally distributed between the electric and magnetic fields $\left(\varepsilon_{r} \varepsilon_{0} E^{2}\right.$ $\left.=\mu_{r} \mu_{0} H^{2}\right)$ and for the dielectric $\varepsilon_{r}=\mu_{r}=1$, then the two components exhibit identical enhancement factors. It is also worth noting that a resonant cavity can also be constructed by using two high index dielectric films $\left(\varepsilon_{r}>0\right)$. Although we do not show this result here, it is easy to see that each mirror will reflect the electric field in phase and hence the opposite positioning of field maxima will be exhibited (i.e., a peak in the electric field at the mirror surface). In this way, high index insulators may be considered as "magnetic" rather than the conventional "electric" mirrors.

If instead, a FP cavity is formed by a plane-parallel insulating slab [Fig. 1(b)] then the resonant field solutions are quite different. Consider a $1 \mathrm{~mm}$ thick slab with relative permittivity and permeability $\varepsilon_{r}=25+0 i$ and $\mu_{r}=1+0 i$, respectively (and hence refractive index $n=\sqrt{\varepsilon_{r} \mu_{r}}=5$ ). In agreement with Eq. (1), the modeled transmission spectra (Fig. 3 inset) suggest that the cavity will support a fundamental mode at a wavelength of $10 \mathrm{~mm}$. The mode exists because a wave inside the cavity will experience a very different impedance $Z$ to a wave outside $\left(Z=Z_{0} Z_{r}\right.$, where $Z_{0}=\sqrt{\mu_{0} / \varepsilon_{0}}$ is the impedance of free space, and $Z_{r}=\sqrt{\mu_{r} / \varepsilon_{r}}$ ) and hence each face of the slab provides a partially reflecting interface. Since the energy stored in the standing wave has to be equally distributed between the electric and magnetic components 


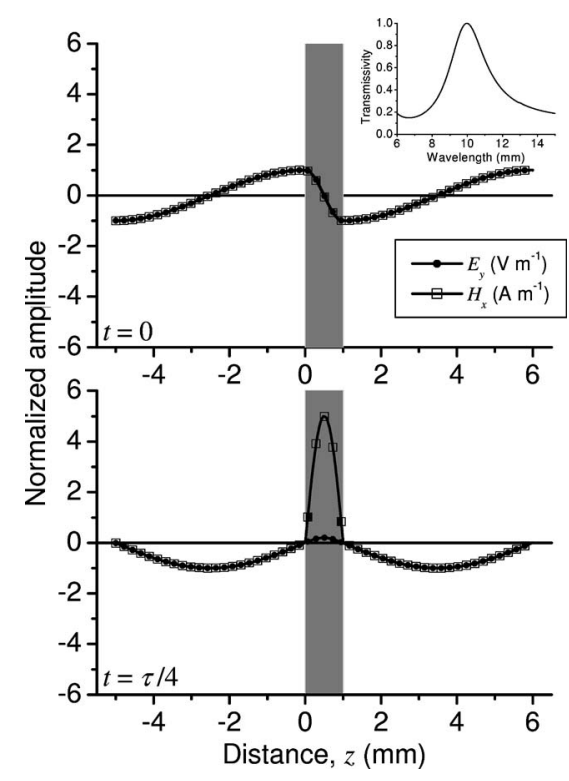

FIG. 3. Predictions of the amplitude of the electric and magnetic fields (normalized to the amplitude of the incident field) through the high index dielectric slab $\left(L_{\text {slab }}=1 \mathrm{~mm} ; \varepsilon_{\text {slab }}=-25+0 i\right)$ structure on resonance of the fundamental mode. Inset: Modeled transmissivity spectrum.

$\left(\varepsilon_{r} \varepsilon_{0} E^{2}=\mu_{r} \mu_{0} H^{2}\right)$, then the ratio of the maximum magnitude of the $H$ field enhancement to that of the $E$ field enhancement is $Z_{r}^{-1}$ (i.e., $=\sqrt{\varepsilon_{r} / \mu_{r}}$ ). Furthermore, no enhancement may occur at the cavity walls due to the requirement to exactly match the electromagnetic boundary conditions (continuous tangential $\underline{\mathrm{E}}$ and $\underline{\mathrm{H}}$ ) at these interfaces. Figure 3 illustrates the modeled resonant field solutions for this cavity, showing that, almost counterintuitively, it is the $H$ field that exhibits an enhancement (by a factor of $n=5$ compared to the electric field). This enhancement must occur at the center of the cavity and be accompanied by a minimum in the magnetic field strength at the walls. Therefore again we can conclude that the resonance is driven by the electric component of the incident field. At a phase $\tau / 4$ earlier, the corresponding peak in the electric field occurs at the walls but without enhancement. Conversely, the resonance of a slab with $\varepsilon_{r}$ $<\mu_{r}$ (not shown) is driven by the magnetic field but would exhibit no enhancement of the $H$ component, while the electric field would show enhancement by a factor of $Z_{r}$ at the cavity center. Clearly a lossless material with $\varepsilon_{r}=\mu_{r}$ supports no resonance, and is totally transparent to radiation (since $Z_{r}=1$ ), even though the refractive index $n$ is finite.

\section{THE METALLIC SLIT ARRAY}

Having examined these two familiar forms of FP resonant cavity, let us now consider a periodic array (pitch $\lambda_{g}$ ) of identical narrow slits (width $w \ll \lambda, \lambda_{g}$ and length $L_{\text {slit }}$ ) cut through a perfectly conducting metal substrate [Fig. 1(c)]. As previously discussed each slit is able to support a TEM waveguide mode that exists without cutoff. ${ }^{2}$ The high metal occupancy of the structure dictates that each face of the slit array will act as a very good, but not quite perfect mirror since weak coupling to the TEM mode will occur. The structure will therefore support standing wave resonances between each face. It becomes highly transmissive at a series of

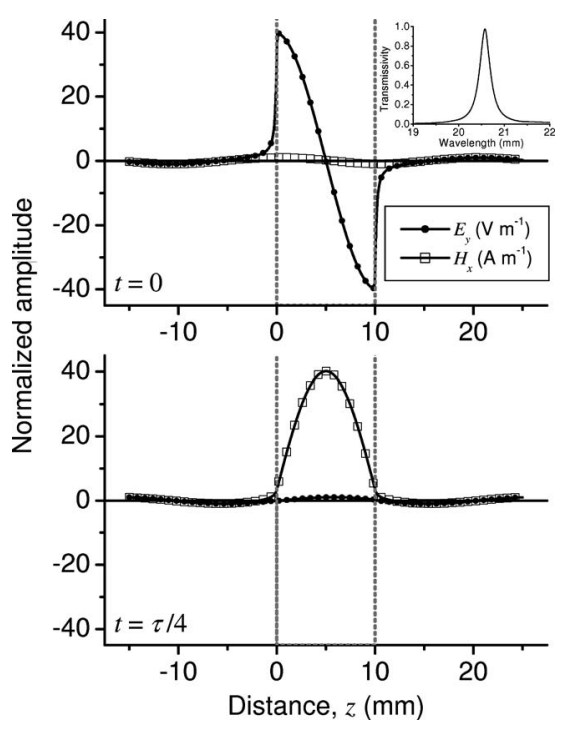

FIG. 4. Predictions of the amplitude of the electric and magnetic fields (normalized to the amplitude of the incident field) along a line at the mid width $(y=w / 2)$ of a slit cavity $\left(L_{\text {slat }}=10 \mathrm{~mm} ; \lambda_{g}=10 \mathrm{~mm} ; w=0.1 \mathrm{~mm}\right)$ in a perfect conductor on resonance of the fundamental mode. Inset: Modeled transmissivity spectrum.

characteristic wavelengths $^{3-8}$ ( of order $N$ ), which in the limit of infinitesimal slit-cavity width are given by Eq. (1) (where $n$ is the refractive index of the material filling the cavities). However, for all real systems, the resonant wavelength exceeds this value due to the finite distance required to match the fields inside the cavity to the free space fields beyond. ${ }^{9,10}$ The inset of Fig. 4 illustrates the modeled transmission spectrum from an array of slits of pitch $\lambda_{g}=10.0 \mathrm{~mm}$, each of width $w=0.10 \mathrm{~mm}$, formed into a perfectly conducting substrate of thickness $L=10.0 \mathrm{~mm}$. Radiation is incident in the $z$ direction, polarized with the electric field vector parallel to the $y$ axis. The $y$ and $x$ components of the electric and magnetic field enhancements, respectively, have been calculated at the resonant frequency of the fundamental mode $\left(\lambda_{N=1}\right.$ $=20.6 \mathrm{~mm}$ ). These are the only two nonzero components of the field at the midplane of the slit cavity $(y=w / 2)$ and are plotted as a function of the distance along the cavity length in Fig. 4. As in the previous discussions, the fields in the upper graph have been calculated at a time in the cycle associated with the maximum enhancement of the electric field. This condition corresponds to the temporal phase at which a peak of the incident electric field occurs at the illuminated face of the substrate $(t=0)$, illustrating that the standing wave modes are again driven by the electric component. Similarly the lower graph shows the same field components one-quarter of a cycle later $(t=\tau / 4)$ corresponding to maximum enhancement of the magnetic field. Notice how, in similarity with the etalon structure, both electric and magnetic components are equally enhanced. However, a comparison of the nodes and antinodes of the field solutions demonstrates that while the etalon exhibits a maximum in the $H$ field at each end of the slit cavity $(z=0$ and $z=L)$ and a maximum in the $E$ field at its midpoint $(z=L / 2)$, the slit array demonstrates a complete reversal of this positioning. The boundary separating the ends of the slit cavities from the semi-infinite space beyond acts as a high-impedance surface, 


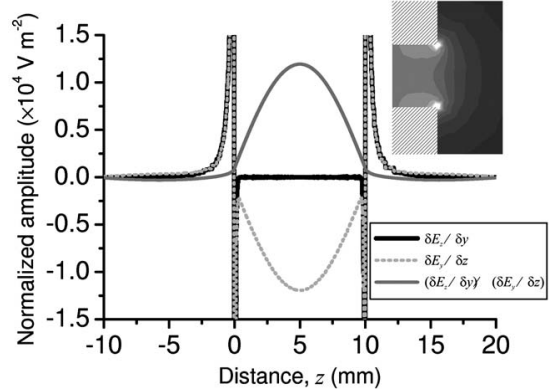

FIG. 5. Predictions of the time-averaged field amplitudes $\delta E_{z} / \delta y$ and $\delta E_{y} / \delta z$ (normalized to the amplitude of the incident field) on resonance of the fundamental mode of the slit array. Also shown is the difference between these two field differentials, which has the same form as $H_{x}$. Inset: Timeaveraged electric field strength around the end of the slit $(z \sim L)$. White regions correspond to field enhancement factors of at least 65 .

rather like the boundary conditions observed at the surface of an artificial magnetic conductor. ${ }^{13,14}$ As such, from inside the slit cavity, these interfaces reflect the electric field without phase change (i.e., the reflection amplitude coefficient is positive) and hence the time-averaged electric field exhibits maxima at these boundaries.

The relative positions of the field maxima in the conventional etalon structure can be easily explained by employing the conventional boundary conditions on the metal mirrors. However there is clearly no equivalent explanation that is applicable for the slit-cavity array; instead the answer lies with the surface currents supported on the walls defining each slit. A longitudinal current-density standing wave is established in the $z$ direction on both of the two metal walls of each slit by the oscillating electric field. At any instance the current flow on one of the metal surfaces must be opposite to that on the other, and hence a net magnetic field is induced in the $x$ direction. The current-density standing waves have their maxima away from the ends of the slit cavities (e.g., at $z=L / 2$ for $N=1$ ) since at their ends current is clearly unable to flow in the $z$ direction. Hence the magnetic field strength of the standing wave mode at both ends of the cavities $(z$ $=0$ and $z=L)$ must also be small and accompanied by a corresponding peak in the electric field strength.

Having drawn conclusions concerning the relative positions of the field maxima within the slit cavities, we have not yet commented on how the matching process between the external propagating waves and the cavity modes occurs. We illustrate in Fig. 4 how $E_{y}$ and $H_{x}$ are the only two nonzero components along the midplane of the slit cavities $(y$ $=w / 2$ ). However this is generally not the case away from this plane. For example, consider how the electric field is strongly diffracted at each end of the slit cavities (Fig. 5 inset) leading to finite $E_{z}$. Furthermore the derivative of $E_{z}$ with respect to $y$ must also be nonzero across its entire width at the ends. Hence at the ends of the slits, with $E_{z}$ and $\partial E_{z} / \partial y$ finite, Maxwell's equation $\nabla \times \underline{\mathrm{E}}=-\mu \partial \underline{\mathrm{H}} / \partial t$ reduces to

$$
\left|\frac{\partial E_{z}}{\partial y}-\frac{\partial E_{y}}{\partial z}\right|=\frac{2 \pi c \mu}{\lambda}\left|H_{x}\right| .
$$

Crucially, $\partial E_{z} / \partial y$ will vary over a distance corresponding to the slit width $(w<<\lambda)$. Therefore at the ends of each of the

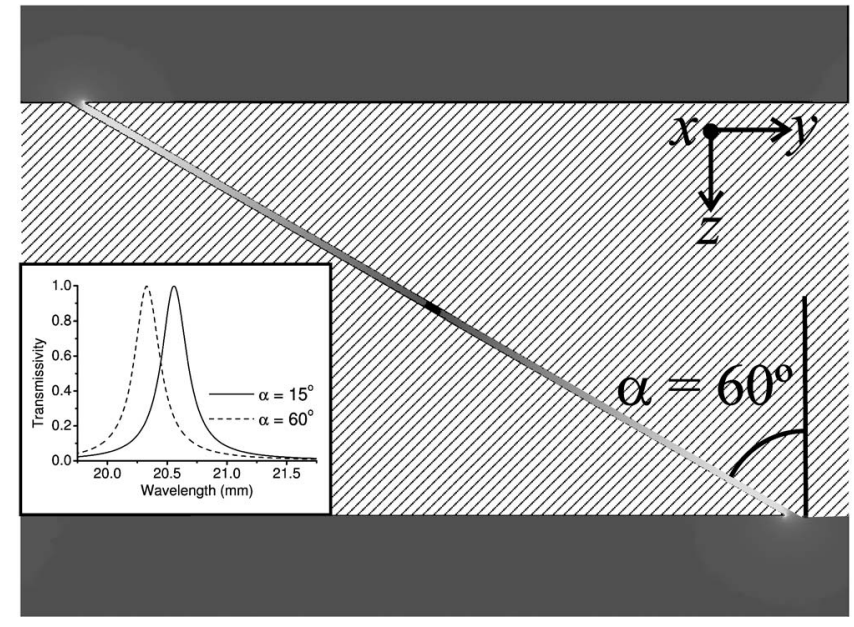

FIG. 6. Time-averaged electric field strength on resonance of the fundamental mode of an array of tilted slit cavities $\left(\alpha=60^{\circ}\right)$. Radiation is incident in the $z$ direction, polarized with its electric vector parallel to the $y$ axis. White regions correspond to field enhancement factors (compared with the intensity of the incident field) of at least 100 (linear scale). Inset: Modeled transmission spectra of an array of identical slits formed at an angle $(\alpha)$ to the surface normal. The angle $\alpha$ is varied while keeping the slit length, width, and pitch fixed.

slits, strong diffraction leads to both $\partial E_{z} / \partial y$ and $\partial E_{y} / \partial z$ being large and approximately equal (Fig. 5). The $\pi / 2$ phase change in $E_{y}$, required to satisfy the matching of the cavity's standing wave field to the propagating free space field beyond, will be compressed into a distance of order $w(\ll \lambda)$. The electric field plot of Fig. 4 illustrates this fully.

Perhaps one of the most significant attributes of the slitcavity array is that the slits do not have to be normal to the face of the substrate. For example, the slit cavities may be tilted at an angle to the surface normal without significantly perturbing the resonant wavelength of the structure provided that the slit length does not change. Figure 6 illustrates the result of forming each of the slit cavities in this way. The device thickness is reduced by a factor of two by tilting the slits to an angle of $\alpha=60^{\circ}$ while only marginally decreasing the resonant wavelength (due to end effects). Furthermore, the slits need not even be straight. This is because the narrow width of the slit cavity ensures that the phase shift associated with the path difference at any corners is a small fraction of the resonant wavelength, and hence the corners do not significantly perturb the fundamental resonant mode expected at $\lambda \approx 2 L$ (where $L$ is the mean cavity length). In this way the sample thickness may be further reduced as a pair of rightangle corners enabling most of the slit length to be directed parallel to the surface. Figure 7(a) shows the resonant fields of the fundamental mode in an array of such slit cavities where the overall thickness of the device is now less than $2 \%$ of the resonant wavelength. Indeed by reducing the pitch so that the upper and lower apertures exactly overlap, an ultrathin device with a continuous core parallel to the sample surface may be envisaged, sandwiched between two metallic metal-slit layers. A standing wave is established along the length of the core, and hence transmission resonances are now quantized by the pitch of the slits $\lambda_{g}$ [Fig. 7(b)]. Such a structure has recently been experimentally demonstrated by the authors and is presented with modeled data and field 


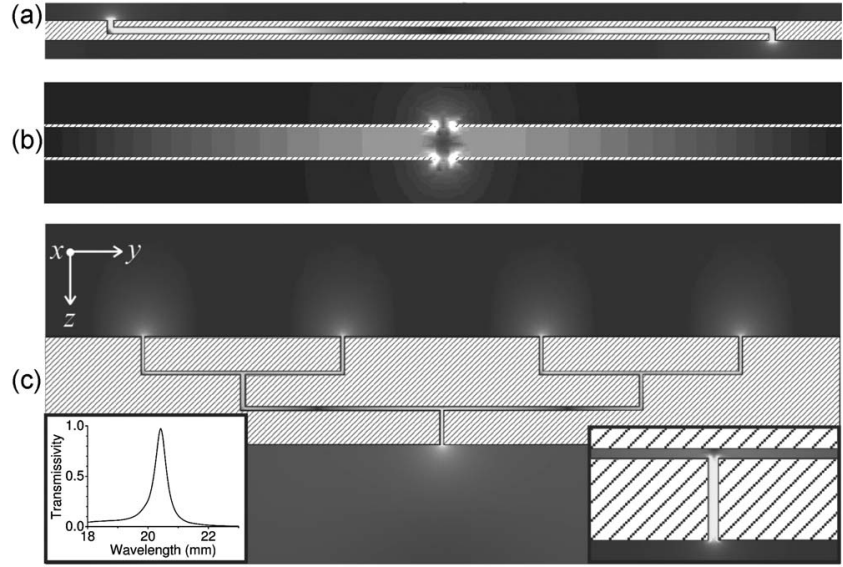

FIG. 7. Time-averaged electric field strength on resonance of the fundamental mode of more complex metallic slit-cavity arrays. For each structure a single unit cell is shown and radiation is incident in the $z$ direction, polarized with the electric vector parallel to the $y$ axis. (a) Total cavity length $=10.0 \mathrm{~mm}$, cavity width $=0.10 \mathrm{~mm}$. White regions correspond to field enhancements of at least 80 times. (b) The structure studied in Ref. 14: pitch $=10.0 \mathrm{~mm}$, cavity (core) width $=0.36 \mathrm{~mm}$, cavity (core) refractive index $=2.05$. White regions correspond to enhancements of 20 times or more. (c) Vertical sections have length of $0.80 \mathrm{~mm}$, the pair of upper horizontal sections has length of $5.00 \mathrm{~mm}$, the lower horizontal length is $10.0 \mathrm{~mm}$, and cavity width $=0.10 \mathrm{~mm}$. White regions correspond to field enhancements of at least 180 times (log scale). Left-hand inset: Transmission spectra. Right-hand inset: An enlargement around the final vertical length of the cavity. The greyscale is linear with white shading corresponding to enhancements of at least 125 times.

predictions in full in Ref. 15. Finally it is also interesting to consider a family of slits, each of the same length, but which merge so that the number of entrance apertures does not equal the number of exit apertures. Consider, for example, an infinite array formed from the unit cell illustrated in Fig. 7(c). The entrances to each of the cavities are spaced by $5 \mathrm{~mm}$ on the illuminated face, with each slit combining with others to give an array of exit apertures with periodicity $20 \mathrm{~mm}$ on the lower surface. Nevertheless, each path length through the substrate is identical $(L \sim 10 \mathrm{~mm})$ and the structure is predicted to exhibit a single strong resonance of the fundamental mode (see left-hand inset) and electric field enhancements of up to 180 times in the final vertical segment. While absorption in real metals will reduce this enhancement factor, these structures may nevertheless be employed for the study of high field induced nonlinear effects. Further, it is important to remember that the periodicity of the apertures on the lower face is much greater than that of those on the upper face. As such this device may be utilized as a diffraction grating that supports no diffracted orders in reflection but does have diffracted orders on resonance in transmission. ${ }^{16}$ In addition, if a unit cell is used in isolation rather than as an array, then this device provides a "radiation funnel," channeling power collected over a large area into a single, bright line source.

\section{CONCLUSIONS}

In this article we have provided a thorough discussion of the resonant fields of the metallic slit-cavity Fabry-Perot resonator, making comparisons to more familiar FP geometries. Our analysis provides an elegant illustration of the application of Maxwellian electromagnetism by demonstrating that the interface between the ends of each slit cavity and the semi-infinite space beyond acts as if it was a high impedance surface, where the boundary conditions are satisfied through the strong diffraction of the electromagnetic field. We have discussed how the slits can be tilted or bent to form samples of thicknesses many times less than their resonant wavelength and can be combined in order to investigate extremely high field enhancements or provide ultrabright line sources.

${ }^{1}$ C. Fabry and A. Perot, Ann. Chim. Phys. 16, 115 (1899).

${ }^{2}$ R. E. Collin, Field Theory of Guided Waves (IEEE, New York, 1991), Chap. 5.

${ }^{3}$ J. A. Porto, F. J. Garcia-Vidal, and J. B. Pendry, Phys. Rev. Lett. 83, 2845 (1999).

${ }^{4}$ S. Astilean, P. Lalanne, and M. Palamaru, Opt. Commun. 175, 265 (2000).

${ }^{5}$ A. P. Hibbins, J. R. Sambles, C. R. Lawrence, and D. M. Robinson, Appl. Phys. Lett. 79, 2844 (2001).

${ }^{6}$ F. Z. Yang and J. R. Sambles, Phys. Rev. Lett. 89, 063901 (2002).

${ }^{7}$ S. Collin, F. Pardo, R. Teissier, and J. L. Pelouard, J. Opt. A, Pure Appl. Opt. 4, S154 (2002).

${ }^{8}$ G. A. Kriegsmann, J. Appl. Math. 65, 24 (2004).

${ }^{9}$ Y. Takakura, Phys. Rev. Lett. 86, 5601 (2001).

${ }^{10}$ J. R. Suckling, A. P. Hibbins, M. J. Lockyear, T. W. Priest, J. R. Sambles, and C. R. Lawrence, Phys. Rev. Lett. 92, 147401 (2004).

${ }^{11}$ HFSS, Ansoft Corporation, Pittsburgh, PA, USA.

${ }^{12}$ E. D. Palik, Handbook of Optical Constants of Solids (Academic, New York, 1985).

${ }^{13}$ D. Sievenpiper, L. J. Zhang, R. F. J. Broas, N. G. Alexopolous, and E. Yablonovitch, IEEE Trans. Microwave Theory Tech. 47, 2059 (1999).

${ }^{14}$ Y. Zhang, J. von Hagen, M. Younis, C. Fischer, and W. Wiesbeck, IEEE Trans. Antennas Propag. 51, 2704 (2003).

${ }^{15}$ A. P. Hibbins, J. R. Sambles, C. R. Lawrence, and J. R. Brown, Phys. Rev. Lett. 92, 143904 (2004).

${ }^{16}$ M. J. Lockyear, A. P. Hibbins, K. R. White, and J. R. Sambles, Phys. Rev. Lett.(submitted). 